\title{
Campañas 2.0 en la Comunidad Valenciana: las primarias en Coalició Compromís y Ciudadanos como estudio de
} caso

institucional.us.es/ambitos/

\section{Marta Pérez Gabaldón}

Universidad CEU-Cardenal Herrera

marta.perez@uch.ceu.es

\section{Blanca Nicasio Varea \\ Universidad CEU-Cardenal Herrera \\ blanca.nicasio@uch.ceu.es}

English Version: Political campaign 2.0 in Valencia community: the primaries in Coalició Compromís and Ciudadanos as a study case

\section{Resumen}

Las redes sociales son un instrumento consolidado en el desarrollo de las campañas pues favorecen la participación ciudadana, la comunicación instantánea y la interacción del político con el usuario-ciudadano. El presente estudio tiene como objeto el análisis de las campañas 2.0 celebradas en Coalició Compromís y

Ciudadanos para elegir, por medio de unas primarias que tuvieron lugar entre diciembre de 2014 y febrero de 2015, a sus respectivos candidatos como representantes de la ciudadanía valenciana en Les Corts. Así, se examina la gestión de Twitter y Facebook de los candidatos y de los partidos, sus web y la respuesta ciudadana.

\section{Palabras clave}

Campañas 2.0, primarias, redes sociales, Compromís, Ciudadanos.

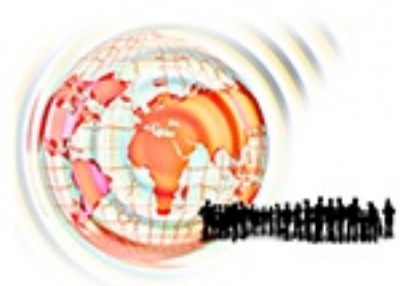

Social media is a consolidated tool in political campaign development. It facilitates citizen participation, instant communication and interaction between the politician and the user/citizen. This study aims to analyze the political campaign 2.0 celebrated within Coalició Compromís and Ciudadanos resulting in the election of their candidates in primaries. Thus, the management of Twitter and Facebook accounts of candidates and political parties, as well as their websites and the citizens' responses are examined.

\section{Keywords}

Political campaign 2.0, primary election, social media, Compromís, Ciudadanos.

\section{INTRODUCCIÓN: MARCO TEÓRICO}

La irrupción de internet supone un desafío para la política, un desafío para los medios de comunicación, y un desafío para la nueva ciudadanía de la democracia, cuya participación en el debate público pasa ahora por la red (Rey Morato, 2007:302 - 303). Sin lugar a duda, la

\section{ÁMBITOS}

2015

$n^{2} 29$ combinación de todo ello incide en el cambio de paradigma de las tradicionales campañas electorales, pues los medios convencionales han cedido el papel protagonista ante los social media que se han posicionado como el canal más rápido y efectivo para hacer llegar el mensaje al votante-usuario.

En la actualidad, los ciudadanos tienen más acceso que nunca a Internet y a los nuevos medios, especialmente a las plataformas de redes sociales. Según la Encuesta sobre Equipamiento y Uso de Tecnologías de 
Información y Comunicación en los Hogares, realizada por el Instituto Nacional de Estadística [1] en 2014, el $74,4 \%$ de los hogares españoles dispone ya de conexión a Internet, casi cinco puntos más que el año precedente y, por primera vez en nuestro país, hay más usuarios de Internet $(76,2 \%)$ que de ordenador $(73,3 \%)$. De los datos que componen la Encuesta se desprende, más concretamente, que el $68,6 \%$ de los valencianos son usuarios frecuentes de las NTICS.

Internet y los medios sociales procuran un nuevo modelo de comunicación horizontal en el que emisores y receptores o, en términos electorales, candidatos y votantes (Sampedro, López y Muñoz, 2012: 659), convergen abiertamente en un mismo espacio. Las posibilidades que ofrecen las nuevas tecnologías de la información y la comunicación (NTICS) para que una fuerza política converse con sus militantes y simpatizantes son enormes. De hecho, es suficientemente hercúlea como para que se plantee la necesidad de olvidar la palabra militante y abrirse hacia una esfera más extensa (Caldevilla, 2009: 32) que tiene como base el ciberactivismo (De Ugarte, 2007: 85). Cada vez más, los políticos tienden a sumar, a la comunicación política tradicional de carácter unidireccional, estos otros canales que requieren de una actitud proactiva, de tal modo que "la clase política tendría que escuchar la conversación y los flujos informativos producidos en estos ambientes y participar" (Abejón et al., 2012: 155). Ello supone no caer en lo que Castellsdenomina "tablón de anuncios digital" (2010: 12); esto es,la práctica de uso de Internet en la que el candidato se limita a hablar de las actividades y la propia campaña electoral, con el objeto de darles la mayor difusión posible, sin generar feedback o posibilidad de interacción con sus seguidores.

La democracia en red permite (al menos en su concepción más utópica) mayor vinculación de la ciudadanía con las decisiones políticas (Sampedro, Sánchez y Poletti, 2013: 108), esto se debe a la naturaleza abierta de las redes que facilita el acceso a la información necesaria y mayor implicación del usuario-votante, lo que en última instancia hace de éste un agente activo del proceso decisor. En este sentido,Corrado y Firestone (1997) pronosticaban, en los noventa, cuatro consecuencias de los nuevos medios de comunicación en el proceso electoral, todas ellas encuadradas en una mejora para la ciudadanía: el fortalecimiento del vínculo de los ciudadanos con los candidatos, la mejora en la información política de los electores, la mayor accesibilidad y visibilidad de los candidatos con menos recursos en el proceso político, y el incremento de las alternativas de los votantes y de la participación cívica. A tal efecto, y por lo que aquí concierne, se puede asimilar el proceso electoral al proceso de primarias en un partido, en tanto en cuanto en éste último también se observa la impronta de tales consecuencias (positivas desde la perspectiva del presente estudio) de los nuevos medios de comunicación.

Se trata, pues, de una nueva forma de hacer política, una nueva forma de plantear y ejecutar las campañas en las que los entornos 2.0 cobran especial relevancia dado que es ahí donde, cada vez más, se desarrolla un diálogo y una interacción social. Las redes, por el volumen de participantes y por las condiciones de control sobre el mensaje, se observan como una herramienta idónea para ganar adeptos en la actividad política, tanto en las planificaciones de las grandes actuaciones de campaña como en la necesaria parcela de comunicación interpersonal que necesita fomentar todo candidato para mantener o crear imagen, para difundir ideas, para fomentar círculos de apoyo, para relacionarse directamente con sus votantes o para marcar distancias sobre sus adversarios (Túñez y Sixto, 2011).

En España, son las elecciones generales de 2008 las que marcan un punto de inflexión, en este sentido (Sampedro, López y Muñoz, 2012; López, Lara y Sánchez-Duarte, 2011,Peytibi, Rodríguez y Gutierrez-Rubí, 2008, entre otros). Los partidos en campaña emplearon, por primera vez, y de manera masiva, las herramientas digitales intentando captar la atención y fomentar la implicación ciudadana. Los candidatos españoles tenían webs personales y participaban en plataformas de intercambio de contenidos como Facebook, Tuenti, Youtube o Flickr, entre otras (Sampedro, Sánchez y Poletti, 2013: 106). Desde ese momento, la ciudadanía ha sido testigo de la introducción de nuevos repertorios de acción y participación social así como de un mapa más complejo de comunicaciones, en el que se integra la dimensión digital (García, 2013: 96) que va adquiriendo un peso mayor no solo en periodos electorales, sino también en el día a día. Ahora bien, ello no obsta para una mayor intensidad de uso en aquellos momentos en los que el político precisa de un respaldo social, ya sea de cara a unas elecciones, ya sea de cara a unas primarias, en las que precisa una movilización de sus seguidores/amigos en un sentido determinado. 


\section{OBJETIVOS Y METODOLOGÍA}

En el contexto apuntado, en la presente investigación se analizan las campañas en los entornos 2.0 celebradas en el seno de Coalició Compromís y Ciudadanos para elegir, por medio de unas primarias que tuvieron lugar entre diciembre de 2014 y febrero de 2015, a sus candidatas como representantes de la ciudadanía valenciana en Les Corts. El objetivo es examinar la gestión de las redes sociales, Twitter y Facebook concretamente, de las candidatas Mónica Oltra [2],de Coalició Compromís, y Carolina Punset [3], de Ciudadanos,y de los partidos [4], así como la web de estos últimos, sin dejar de lado la necesaria aproximación a la respuesta ciudadana ante la demanda de participación por parte de las fuerzas políticas en sus procesos democráticos internos.

La selección de los partidos y sus candidatas se basa en los siguientes elementos en términos acumulativos. En primer lugar, se optó por analizar partidos no tradicionales y/o de reciente creaciónen tanto en cuanto "los partidos nuevos y pequeños son más propensos a explotar los nuevos mecanismos de comunicación ofrecidos por Twitter" (Aragón, Kappler, Kaltenbrunner, Laniado y Volkovich., 2013: 202) y otras redes sociales, realidad que llevó a no incluir en el estudio a Partido Popular, Partido Socialista Obrero Español e Izquierda Unida. En segundo lugar, se atendió, dentro de estos, a aquellos partidos con expectativas de obtener representación en el parlamento autonómico, atendiendo a las encuestas pre-electorales, motivo por el que se descartó incluir a Unión, Progreso yDemocracia (CIS, marzo 2015). En tercer y último lugar, se consideró esencial que los candidatos tuviesen previamente un cargo de representación política en la Comunidad Valenciana, realidad que llevó a prescindir de Podemos, y centrar el estudio en Ciudadanos, cuya candidata fue concejal en el Ayuntamiento de Altea por Ciudadanos independientes por Altea, y Compromís, cuya candidata ha sido diputada de Les Corts en las dos legislaturas precedentes.

A partir de las investigaciones sobre los cambios que se producen en los procesos de comunicación política relacionados con el desarrollo de Internet y los medios sociales, planteamos como hipótesis:

H1. Cabe esperar, en coherencia con estudios previos (Aragón, 2013; Zugasti y Pérez, 2015; Congosto, 2015, entre otros) un uso determinante de los medios socialesy la web como canales de comunicación para informar sobre la campaña.

H2. Se espera hallar por parte de los partidos una tendencia al recurso de los medios socialespara generar y difundir contenido sobre las primarias, en especial, de apoyo a las candidatas así como de movilización a los usuarios para que participen en la votación (Abejón et al., 2012: 131).

SH2.1: Cabe esperar que, dada la mayor brevedad de la campaña de Ciudadanos, este publicará más contenido en sus perfiles en redes sociales.

H3. Se prevé que la utilización de las redes sociales por parte de las dos candidatas sea distinta. Cabe esperar diferencias en cuanto al contenido publicado en relación a la campaña, a la actualización de la misma y a la respuesta de los usuarios. De la anterior hipótesis, se pueden plantear las siguientes subhipótesis:

SH3.1: Atendiendo a estudios previos que demuestran el uso predominante de los medios sociales por parte de los candidatos situados a la izquierda del espectro político y, especialmente, por aquellos que están en la oposición al Gobierno (Vergeer, Hermans ySams, 2011), cabe hallar una mayor proactividad en los perfiles 2.0 de la candidata de Coalició Compromís, Mónica Oltra, respecto a la candidata de Ciudadanos, Carolina Punset.

SH3.2: Dada la mayor trayectoria en redes sociales y el mayor número de seguidores, cabe esperar mayor respuesta e interacción ciudadana en los perfiles de Mónica Oltra. En Twitter, en relación al número de retuits y favoritos y en Facebook, en la cantidad de me gusta, de comentarios y de veces compartido.

Para dar respuesta a las hipótesis y subhipótesis de investigación, analizamos, de un lado, los mensajes publicados en el perfil de Mónica Oltra y de Compromís, en las redes sociales Twitter y Facebook. De otro lado, los mensajes publicados en el perfil de Carolina Punset y de Ciudadanos, en los mismos medios sociales; así como las páginas web de los partidos y la respuesta ciudadana 2.0. 
Dado que se pretendeexaminar y analizar la gestión de las redes sociales en la campaña, se han seleccionado, específicamente, los mensajes publicados durante los días que duró la misma. En el caso de Compromís, desde el 29 de diciembre hasta 1 de febrero (los días 27 y 28 de enero se efectuó la votación online y el 31 la presencial). Y, en el caso de Ciudadanos, desde el 31 de enero hasta el 8 de febrero, siendo el 7 el día en el que se deberían haber celebrado las votaciones. En ese sentido, se ha incluido, en el análisis, el día posterior a la votación con el fin de examinar losmensajes referentes a los resultados.

Cabe señalar que, mientras Compromís sí celebró la elección aun teniendo una única candidata para la Presidencia de la Generalitat [5], la votación en Ciudadanos no llegó a realizarse argumentando que Carolina Punset fue la única que obtuvo los avales necesarios para ser candidata en las primarias (logró 647 avales frente a los 103, correspondientes al $10 \%$ del censo de militantes, que precisaba). Por ello, el día 2 de febrero el líder del Partido, publicó un tuit informando de la "elección" de Punset y convocó una rueda de prensa para presentarla públicamente. No obstante, se ha considerado igualmente interesante estudiar el período que abarca desde el inicio de la campaña hasta el día siguiente a la fecha fijada para la votación a fin de ver el uso en redes en relación a las primarias.

\section{Ilustración 1. Tuit de Albert Rivera confirmando a Carolina Punset como candidata a la}

Presidencia de la Generalitat por Ciudadanos.

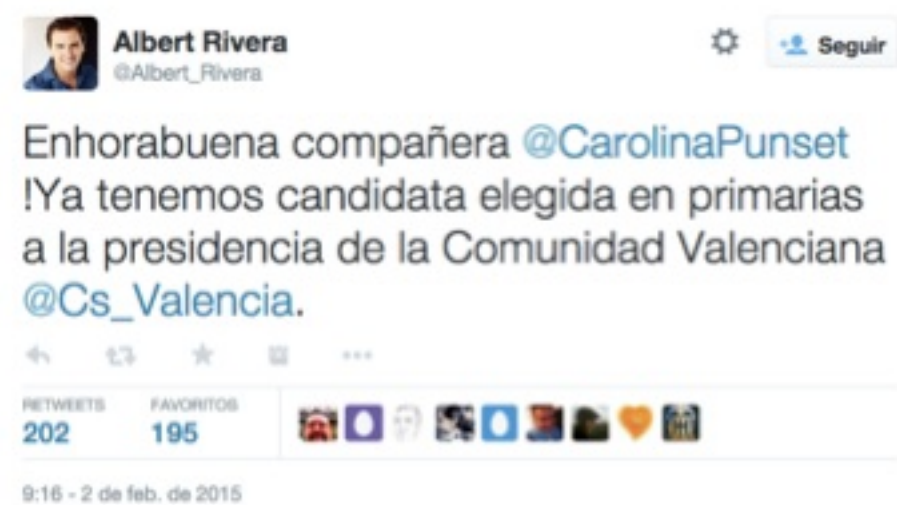

\section{Fuente: www.twitter.com}

El estudio de los tuits se ha realizado en dos niveles, uno cuantitativo y otro cualitativo. Para el análisis cuantitativo se ha recopilado y contabilizado manualmente el número total de tuitsen el período indicado ut supra. En los mensajes propios de las candidatas y de los partidos, además, se ha registrado: el número total de retuits y el número total de favoritos.Para el análisis cualitativo del contenido de los mensajes publicados en los perfiles de las candidatas se han tenido en cuenta las siguientes variables: actos de campaña, petición de participación, petición de voto, información sobre el estado de las primarias (número de inscritos, resultados, días de votación, forma de votación, respaldo a otros candidatos e información relativa al resultado de actos de campaña), propuestas, noticias sobre la campaña en medios de comunicación, apoyo a la candidata, apoyo al trabajo desarrollado durante la legislatura, críticas a otras fuerzas políticas, y otros (en esta variable codificamos aquellos mensajes que no respondan a las anteriores). De ellas, se señala una tras la lectura completa del mensaje de manera que se trata de variables excluyentes.

Para el análisis cualitativo del contenido de los mensajes publicados en los perfiles de los partidos se han utilizado las siguientes variables: actos de campaña, petición de participación y voto, información sobre el estado de las primarias, propuestas, noticias sobre las primarias en medios, información referente al partido,críticas a otras fuerzas políticas, información específica sobre las candidatas y otros.

El estudio de las publicaciones en Facebook, se ha realizado, igualmente, tanto desde el punto de vista cuantitativo como cualitativo. En el análisis cuantitativo se ha contabilizado el número total de mensajes publicados así como la fecha de publicación y el número de me gusta, de comentarios y de veces compartido. Para el análisis cualitativo del contenido de los mensajes se han tenido en cuenta las variables anteriormente descritas. 
Dada la duración desigual de ambas campañas, se ha optado por calcular la media aritmética de mensajes publicados por día en cada una de las cuentas analizadas y de cada una de las vías de respuesta del usuario ofrecida por la red correspondiente. Esto se ha hecho con el fin de poder llevar a cabo un análisis comparado de la frecuencia de uso de las redesy de la respuesta ciudadana respectivamente.

\section{RESULTADOS DE LA INVESTIGACIÓN}

\subsection{Análisis de los resultados globales}

Tras la recopilación de la información apuntada en el apartado anterior, se ha registrado un total de 412 tuits(el $40 \%$ son propios y $60 \%$ retuits) y 96 publicaciones por parte de Compromís en los 36 días analizados; y 241 tuits(el $54,8 \%$ son propios y $45,2 \%$ retuits) y 31 publicaciones por parte de Ciudadanos en los 9 días objeto de estudio. En términos comparativos, se observa que mientras el primero tiene una media de mensajes diarios de 11,4 en Twitter y 2,6 en Facebook, el segundo muestra una media superior al contar con 26,7 y 3,4 respectivamente tal y como evidencia el Gráfico 1.

Gráfico 1: media diaria de publicaciones en redes sociales de los partidos.

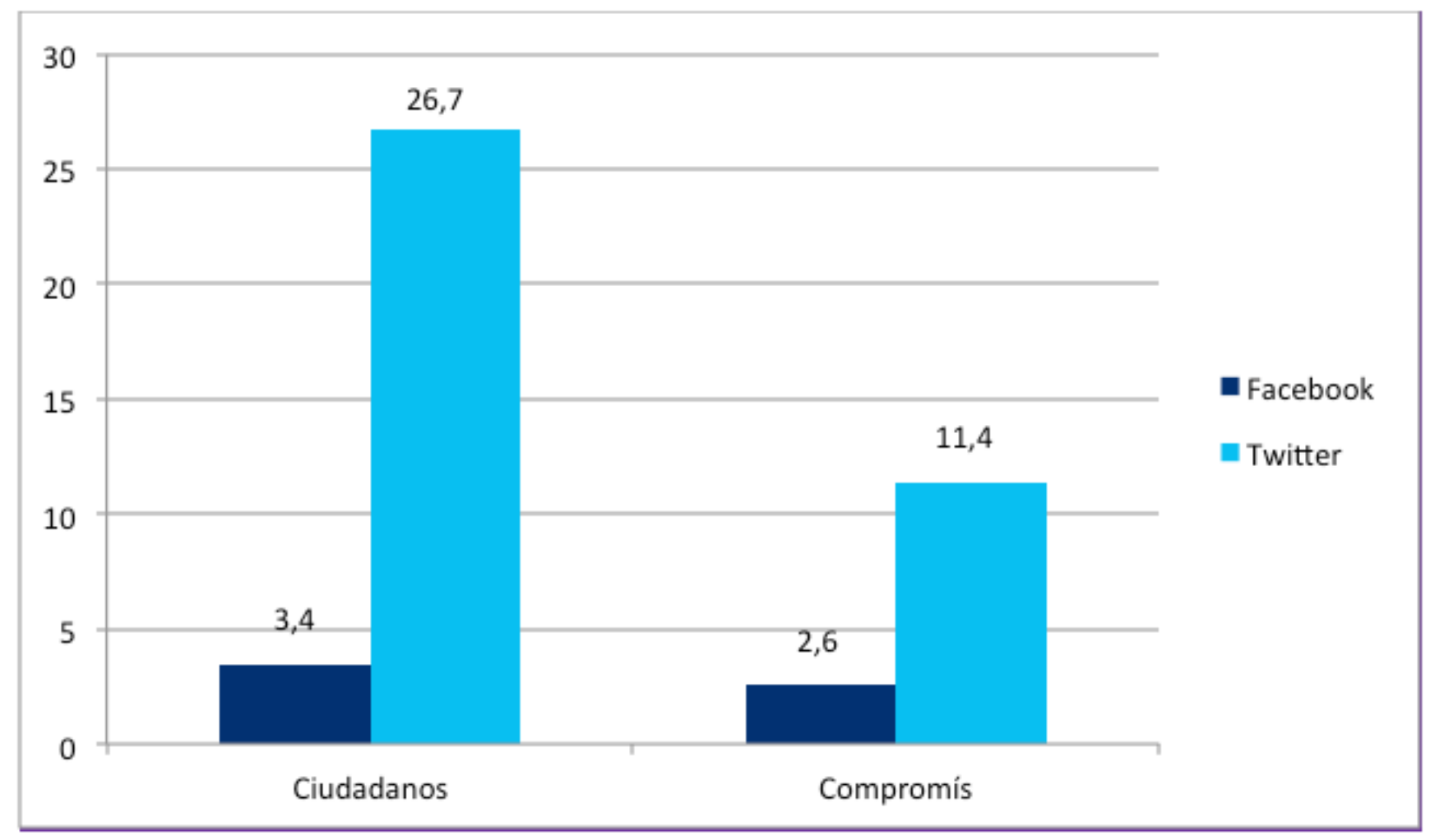

Fuente: elaboración propia

De igual modo, al analizar los datos apuntados, se aprecia una notable preferencia por el uso de Twitter en ambas formaciones, cuestión que avala investigaciones previas (Rodríguez y Ureña, 2011), si bien se ha detectado una tendencia a duplicar la información en las dos redes sociales. Esto podría justificarse por la voluntad de hacer llegar a un mayor número de personas la información generada, aunque también puede provocar una reiteración de contenidos que sobrecargue al seguidor en ambos entornos 2.0.

Por lo que respecta a los perfiles de las candidatas, durante el período analizado, se ha hallado un total de 357 tuits (20\% propios y $80 \%$ retuits) y 44 publicaciones en los perfiles oficiales de Mónica Oltra y 23 tuits (40\%propios y $60 \%$ retuits) y 13 publicaciones en las cuentas verificadas de Carolina Punset. En términos comparados, esto supone que la candidata de Compromís tiene una media diaria de 9,9 mensajes en la red de microblogging y 1,2 en Facebook, mientras que la de Ciudadanos alcanza una media de 2,5 y 1,4 respectivamente. Esto queda reflejado en el Gráfico 2. 
Gráfico 2: media diaria de publicaciones en redes sociales de las candidatas

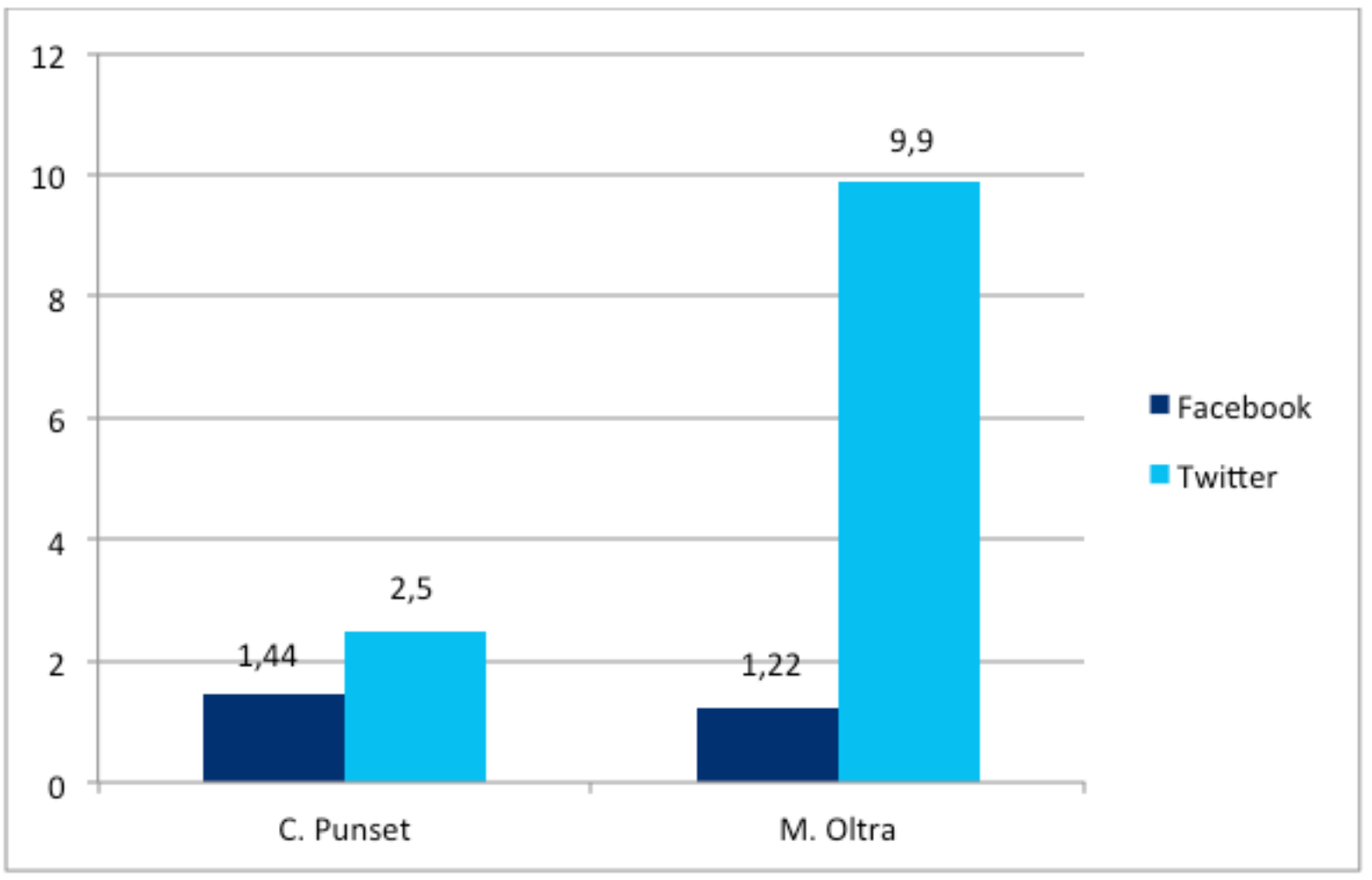

Fuente: elaboración propia

De nuevo, resulta evidente que las candidatas son más proclives a actualizar con mayor asiduidad sus perfiles en Twitter, especialmente Mónica Oltra. Como contrapartida, cabe destacar el escaso uso que la candidata de Ciudadanos hace de los medios sociales, si bien su uso medio de Facebook es superior al de la candidata de Compromís.

En definitiva, siguiendo la estela de un estudio sobre el uso de Twitter en las elecciones europeas de 2009 (VergeerHermans y Sams, 2011), se observa un uso predominante de este medio social por parte de Mónica Oltra en tanto que en ella convergen las características que identificaban a los perfiles más activos en dicho estudio: de un lado, proviene de uno de los partidos situados a la izquierda del espectro político y, de otro lado, la fuerza política a la que representa se encuentra enla oposición al Gobierno desde las elecciones autonómicas de 2007.

El análisis de los portales Web de ambas fuerzas políticas arroja diferencias sustanciales en el tratamiento de las primarias. Compromís tiene una categoría propia denominada "Campañas" dentro de la cual hay un apartado dedicado a las Primarias 2015. Este apartado recoge información sobre los resultados; las candidaturas a Presidencia de la Generalitat, así como a las listas para Les Corts y para los ayuntamientos; el calendario de las primarias; donaciones para la campaña de primarias; y preguntas frecuentes sobre el proceso. Además, se han creado widgets que vinculan el contenido generado en con el hashtag \#primàriescompromís a la Web y, así mismo, redirecciona el tráfico web a la página PrimàriesCompromís de Facebook. A esto se suma la creación del correo electrónico primaries@compromis.net. Esto demuestra el interés del Partido por interconectar sus canales online de relación con el ciudadano y ofrecer diversas vías de participación. 


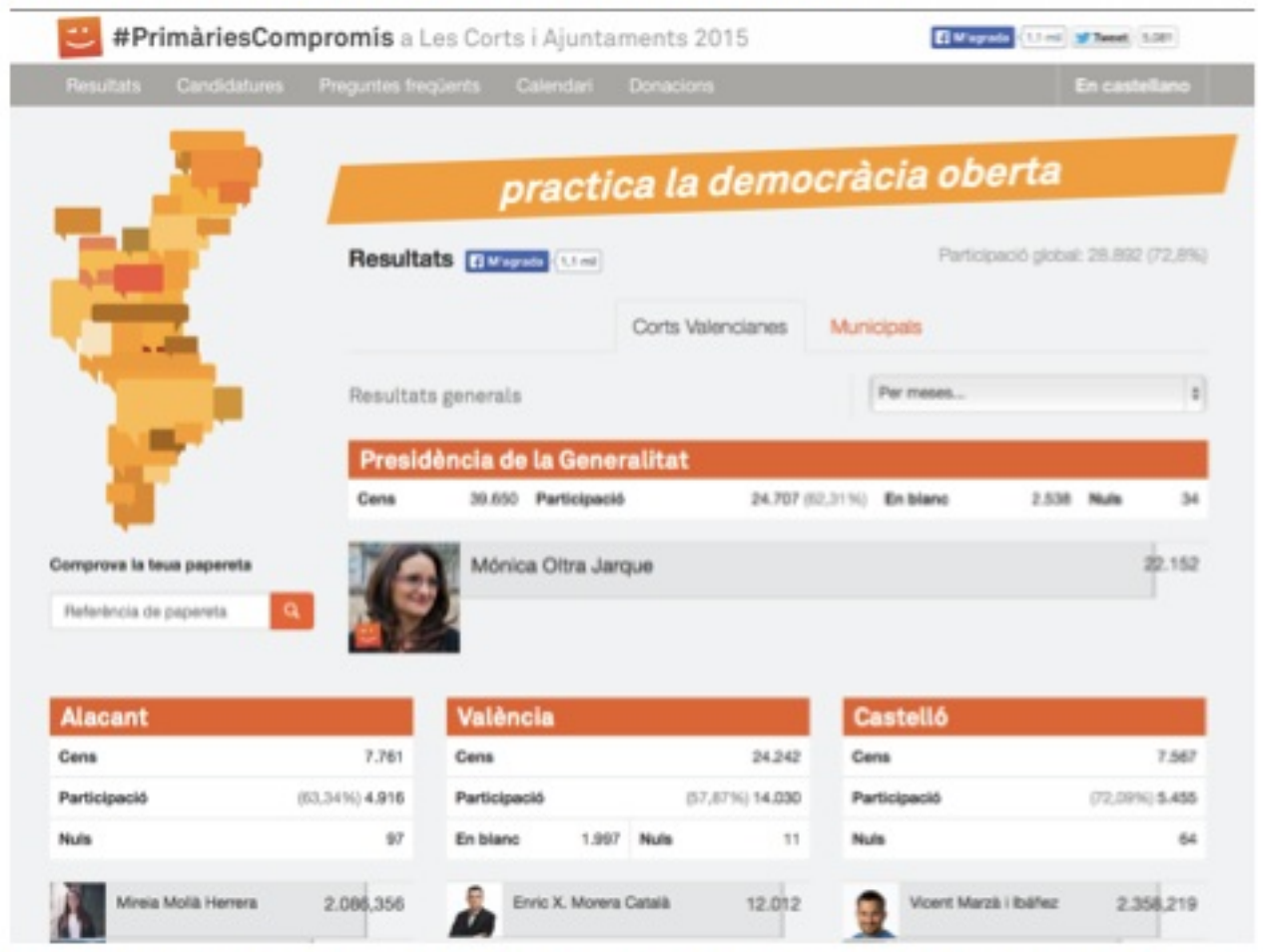

Fuente: www.compromis.net (captura de pantalla realizada el 25 de marzo de 2015)

Por su parte el portal de Ciudadanos, partido de ámbito nacional desde fecha reciente y no exclusivamente autonómico como sucedía en el caso anterior, no contempla ninguna categoría específica en relación a las primarias que, para distintos ámbitos electorales, se han celebrado en el seno del Partido. A esto cabe sumar que tampoco hay un portal propio del Partido en la Comunidad Valenciana, limitándose al uso de redes sociales para la generación y difusión de contenido.

\subsection{Análisis de los perfiles de los partidos}

El objeto del presente apartado es examinar el contenido de los mensajes en entornos 2.0 en base a las variables preestablecidas en la metodología con un doble fin. De un lado, analizar comparativamente cuál es el porcentaje de mensajes relacionados con la campaña y, de otro lado, ver el reparto de dicho porcentaje entre las diferentes variables consideradas en relación a la campaña.

Los perfiles en Twitter de los Partidos dedican una desigual atención a las cuestiones relacionadas con la campaña. Se observa que mientras Compromís dedica un $44 \%$ de sus mensajes, Ciudadanos apenas ocupa con esta cuestión el $23 \%$ del total. Fuera de las cuestiones relacionadas con las primarias, llama la atención el volumen de críticas realizadas, especialmente al partido gobernante, por parte de Compromís (22\%) y el elevado porcentaje de tuits(60\%) que Ciudadanos lanza con información sobre el Partido, incluyendo un buen número de referencias sobre este a nivel nacional o en otras regiones. Si bien es lógico que se emplee el perfil para emitir contenido sobre el Partido a nivel nacional, el perfil relativo a la formación en la Comunidad debería centrar más su atención en su ámbito de actuación, así como en las primarias que estaban llevando a cabo.

Respecto a la cuestión que centra la atención mayoritaria de entre lo relacionado con las primarias, Compromís destaca esencialmente la información sobre el estado de las primarias (15\%), esto es, el número de inscritos, la evolución de los resultados, cómo y cuándo se puede votar, etc. Por su parte, Ciudadanos presta mayor atención a la candidata (15\%), dando información sobre apariciones en medios, entrevistas, actos y demás cuestiones, con el objeto de acercar a Carolina Punset a la ciudadanía valenciana dado que se trata de una política prácticamente desconocida hasta el momento en la Comunidad más allá del ámbito local en el que hasta dicho momento se desenvolvía. 
comentado anteriormente. Esto contrasta con el 13\% de los tuits dedicados a esta cuestión por parte de Compromís, evidenciando con ello la importancia dada por el Partido al proceso y a la transparencia del mismo.

Gráfico 3. Contenido del perfil en Twitter de los partidos políticos (expresado en \%)

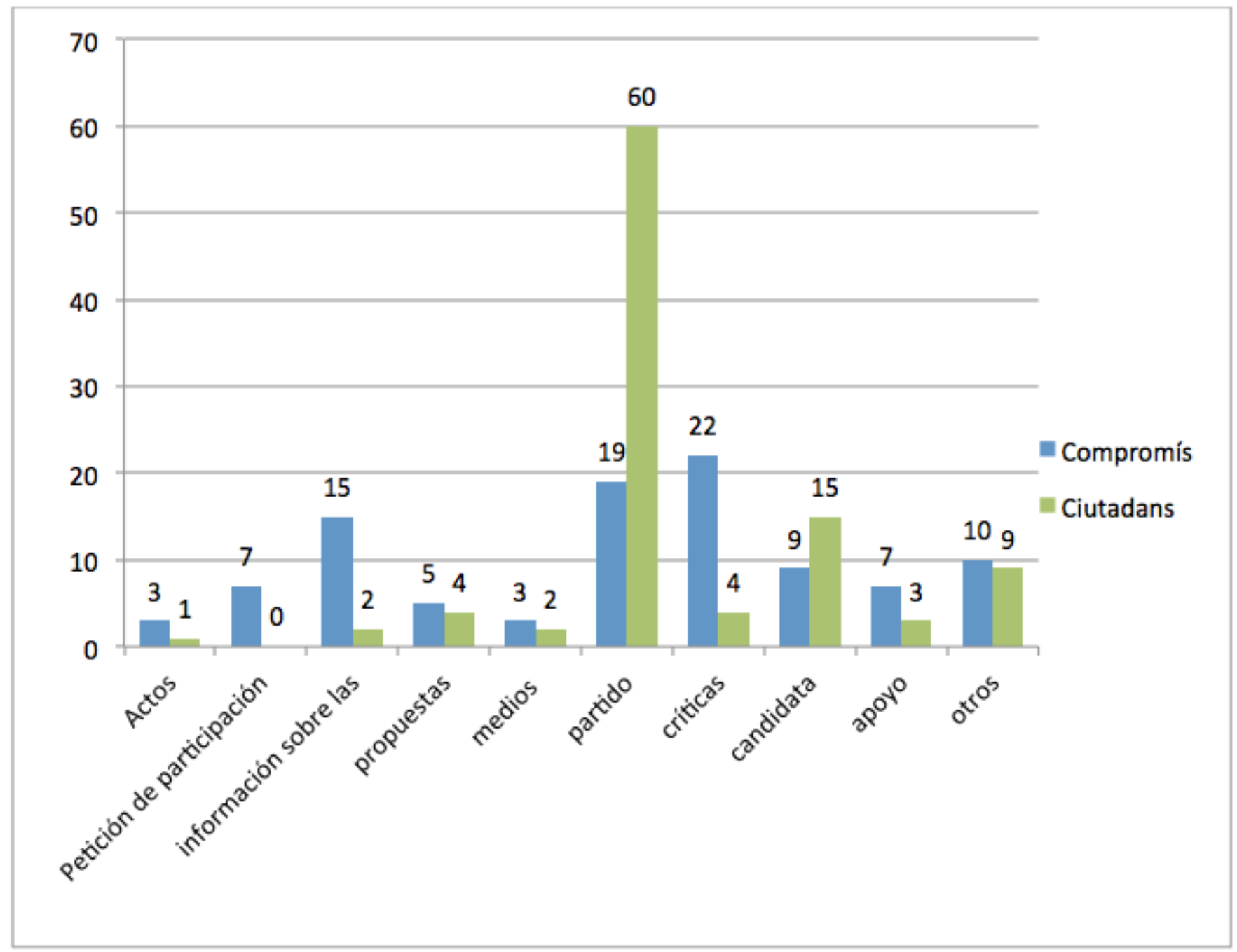

\section{Fuente: elaboración propia}

En los perfiles en Facebook, por su parte, Ciudadanos cuenta con un $42 \%$ de publicaciones relacionadas con las primarias, dándole, por tanto, mayor importancia que en la red de microblogging. De este porcentaje, los actos de campaña y la información sobre Carolina Punset copan un $16 \%$ cada uno, lo que refleja que, al igual que en Twitter, hay una evidente voluntad de dar a conocer y aproximar a la candidata.

En el caso de Compromís, se dedica a las primarias el $41 \%$, siendo la información sobre la candidata el porcentaje más elevado (14\%). Esto muestra una similar dedicación en ambas redes sociales a las primarias por parte del Partido, así como también un porcentaje próximo por parte de ambas fuerzas. Del mismo modo, tanto Ciudadanos como Compromís, centran su atención en difundir información sobre las candidatas.

Tal y como sucedía en Twitter, la cuestión ajena a las primarias que centra su atención es fundamentalmente la generación de información in genere sobre el partido, en el caso de Ciudadanos (48\%), y la crítica al resto de fuerzas políticas (31\%), esencialmente a la que ocupa el gobierno nacional y autonómico, en el caso de Compromís. 
Gráfico 4. Contenido del perfil en Facebook de los partidos políticos (expresado en \%)

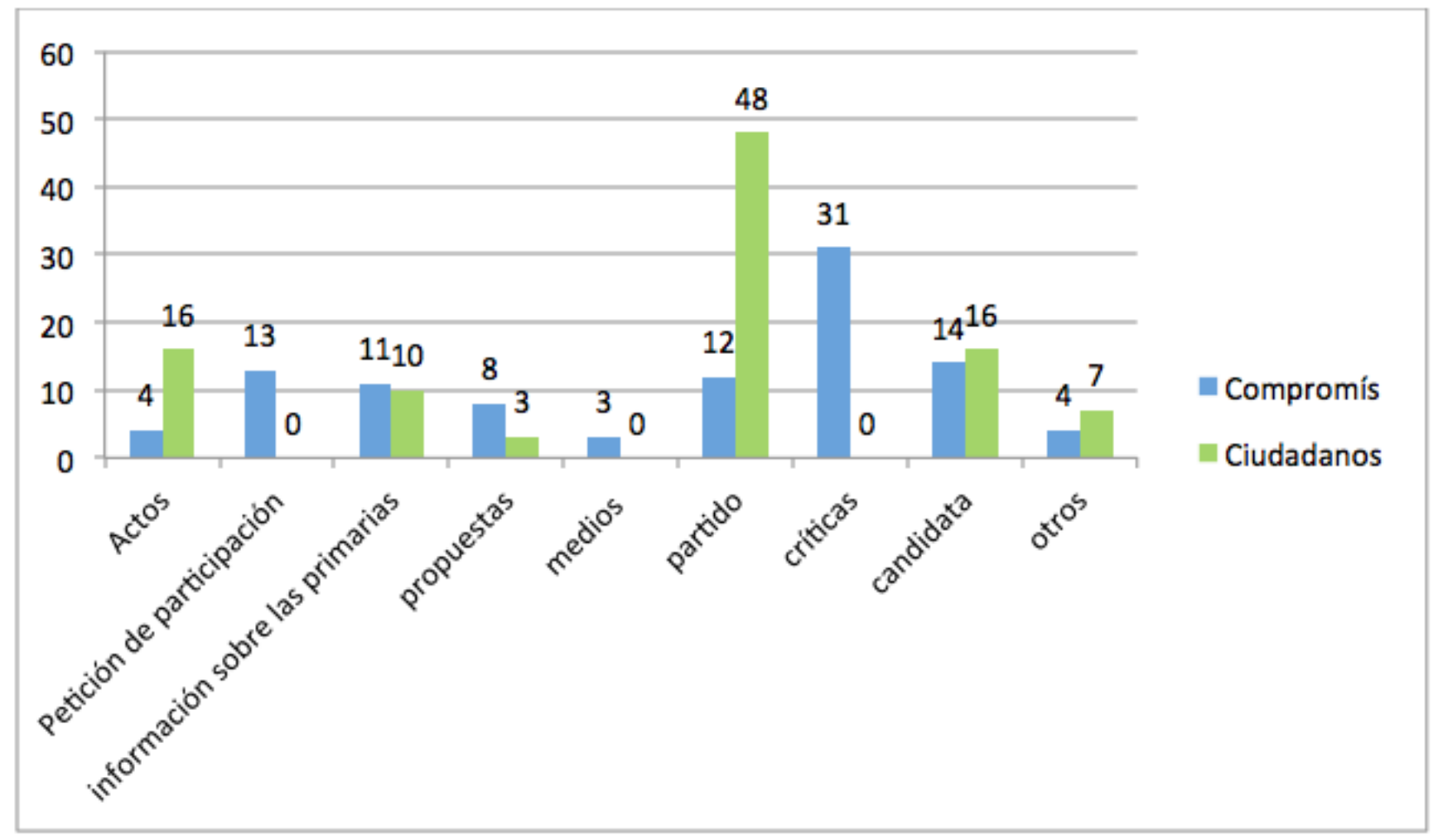

Fuente: elaboración propia

\subsection{Análisis de los perfiles de las candidatas}

Una vez estudiados los perfiles de las fuerzas políticas, cabe centrar la atención sobre las cuentas en las redes de Mónica Oltra y Carolina Punset. De igual modo, el objeto es examinar comparativamente, por un lado, el contenido generado en torno a las primarias y, por otro lado, dentro de este, averiguar qué variable de las analizadas adquiere mayor relevancia para la candidata.

En la red de microblogging, Punset tiene un $56 \%$ de publicaciones relacionadas con las variables de análisis catalogadas como propias de las primarias. No obstante, cabe considerar que no menciona las palabras "primarias", "primaries" o "primàries" en ningúnmomentoy que, en las ocasiones en las que aparece en los medios (13\%) el eje de su intervención no es las primarias, así como también se ha considerado dentro de los actos de campaña (17\%) aquellas apariciones públicas de Punset aunque no fuesen propiamente orientados a pedir el voto o a presentar su candidatura en tanto en cuanto finalmente, como se ha referido anteriormente, no hubo votación. Esta realidad demuestra que se deben contemplar los datos muy estrechamente relacionados con el contexto en el que se desenvuelve el proceso que finaliza antes de hacerlo el plazo establecido para las primarias.

Oltra, por su parte, tiene un $51 \%$ de mensajes relacionados con las primarias. Dentro de este porcentaje, destacan los mensajes de apoyo de ciudadanos que ella, por regla general, retuitea (17\%). Estos mensajes muestran la interacción de ésta con los valencianos que la apoyan para ser la candidata a la Generalitat por Compromís, mientras que no hay ningún retuit de Punset de mensajes de apoyo ciudadano. Por su parte, Oltra sí menciona los conceptos "primarias", "primaries" o "primàries" en un total de 62 ocasiones. 


\section{Gráfico 5. Contenido del perfil en Twitter de las candidatas (expresado en \%)}

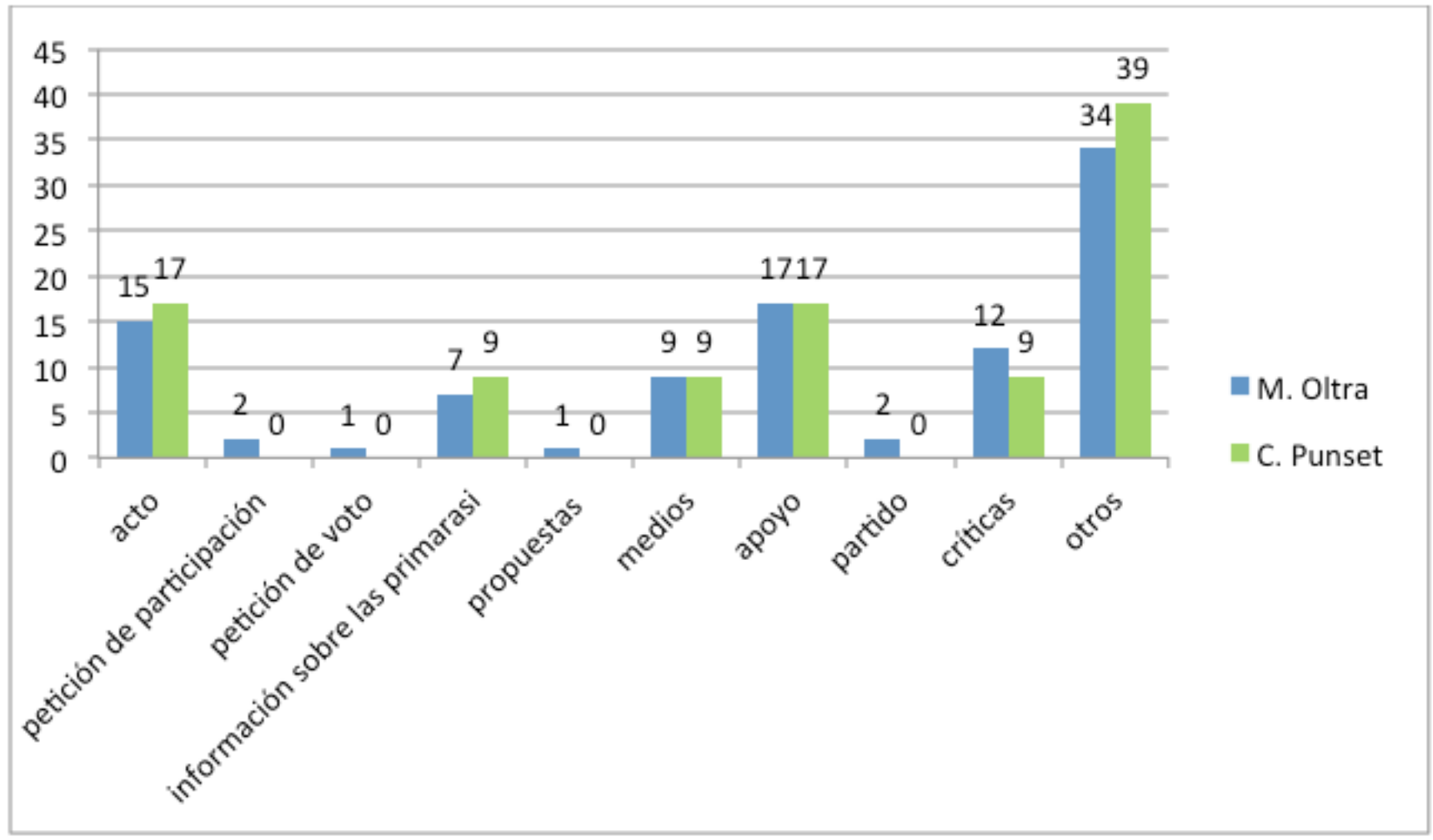

\section{Fuente: elaboración propia}

En el caso de Facebook, el uso realizado por las candidatas resulta de todo punto dispar. Así, mientras Carolina Punset dedica un $61 \%$ de sus publicaciones a las primarias, no repitiéndose los matices realizados en relación a sus mensajes en twitter, Mónica Oltradedica el $79 \%$ del contenido de página oficial a temas relacionados con las primarias. Es este sentido, cabe señalar que la candidata de Compromís utiliza el concepto "primarias", "primaries" o "primàries" en doce ocasiones mientras que la candidata de Ciudadanos no hace uso de estos conceptos en ninguna de sus publicaciones.

Dentro de los temas relacionados con las primarias a los que refieren las candidatas en sus perfiles de Facebook, Oltra da mayor importancia a la promoción de los actos realizados o que va a realizar (39\%), mientras Punset dedica especial atención a informar sobre las primarias y su elección como candidata (23\%) y a sus apariciones en medios de comunicación (23\%). 


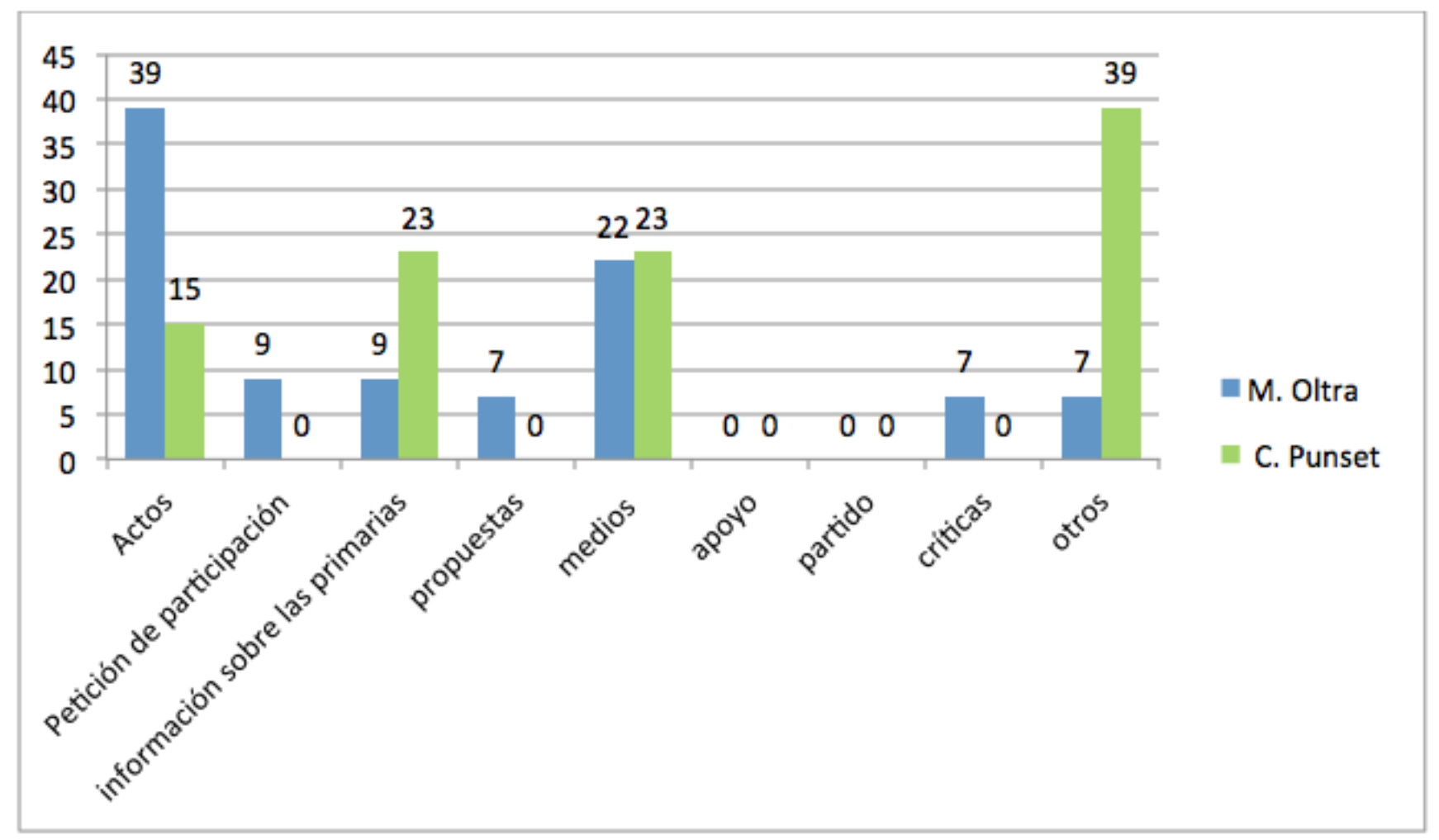

\section{Fuente: elaboración propia}

\subsection{Análisis de la participación y respuesta ciudadana}

Por lo que respecta a este último apartado, se parte de la consideración de que los usuarios utilizan las NTICS para participar en la producción y difusión de la información y en los procesos políticos. De este modo, se ve alterada la comunicación centralizada y verticalista y se favorecen tanto la interacción como las actividades "bottom-up" (Nicasio Varea y Pérez Gabaldón, 2015). Los entornos abiertos favorecen un entorno participativo donde el receptor se torna un agente activo del proceso comunicativo, puesto que "busca, investiga, enlaza, opina, contesta, contrasta y crea contenidos a partir de otros ya existentes" (Caldevilla, 2009: 33), así como también se posiciona mediante el uso de otros medios facilitados por las redes sociales que le permiten mostrar su conformidad y proximidad con los contenidos difundidos por otros usuarios (véase el "me gusta" en Facebook o el "favorito" en Twitter, póngase por caso).Por tal motivo, se ha considerado oportuno analizar, de un lado, como muestra de esa relación ciudadano-candidato y ciudadano-partido, las vías de las que disponen las redes sociales para permitir la interacción de un usuario con otro a partir de un mensaje publicado por uno de ellos.De otro lado,el número de seguidores de los respectivos perfiles como reflejo tanto del alcancereal que un mensaje puede tener entre sus seguidores como de la hipotética difusión que podría alcanzar por el uso de tales vías de interacción.

En relación a la primera cuestión, se ha tenido en cuenta el número total y la media por publicación en el muro de: "me gusta", comentarios y veces compartido de cada mensaje publicado en Facebook en los cuatro perfiles. Asimismo, se ha contemplado el número total y la media por mensaje propio en Twitter de retuits y favoritos.

De las tres vías de interacción facilitadas por Facebook, se observa un número total y una media superior en todos los perfiles analizados de "me gusta", seguido de "compartir", y un número mucho más reducido de comentarios, tal y como puede observarse en la tabla. Esta realidad coincide con estudios previos sobre el uso de esta red y la preferencia del usuario por el like como principal vía de interacción (Merino, Lloves y Pérez, 2013: 865 y 866), vía que puede considerarse de menor grado de implicación que las otras dos. La confrontación de las medias muestra una mayor interacción social con el perfil de Mónica Oltra, contando con una media de 209,09 me gusta, 5,79 comentarios y 44,81 compartidos, frente a los 19,6likes, 1,15 comentarios y 2 compartidos de Punset.Al comparar las medias de Compromís y Ciudatans, se observa el mismo patrón apuntado, de modo que el primero tiene mejor promedio en los tres ítems. 
En el caso de Twitter, sin embargo, se observa un cambio de tendencia puesto que, aun y cuando en términos absolutos Compromís y Oltra tienen mayor número de retuits y favoritos, al observar la media de estos por mensaje propio en los 4 perfiles, los números de Ciudadanos y Punset son mejores.

Respecto a la segunda cuestión, se ha tenido en cuenta el número de seguidores a fecha de cierre dela recopilación de datos para el presente artículo [6]. A tal efecto, cabe destacar, en primer lugar, que la página oficial de Mónica Oltra en Facebook es la que cuenta con un mayor número de "me gusta" y, en segundo lugar, que las candidatas poseen una cantidad de "me gusta" superior al de sus partidos. Además, se observa que el Facebook de Compromís tiene ocho veces más "me gusta" que el de Ciudadanos, y Oltra tiene seis veces más "me gusta" que Punset en dicha red social. Esto puede encontrar su causa en la afianzada trayectoria política en la Comunidad de Oltra y su Partido.

En Twitter, se aprecia, al igual que en Facebook, que, de los cuatro perfiles analizados, el de Mónica Oltra es el que cuenta con un mayor número de seguidores y que las candidatas poseen un número de seguidores en la red de microblogging superior al de sus respectivas formaciones políticas, especialmente en el caso de Oltra.

Tabla 1. Interacción con la ciudadanía en los perfiles analizados

\begin{tabular}{|c|c|c|c|c|}
\hline \multicolumn{5}{|c|}{ Facebook } \\
\hline & Compromis & Ciudadanos & M. Oltra & C. Punset \\
\hline $\begin{array}{l}\text { Seguidores } \\
\text { (me gusta) }\end{array}$ & 27.414 & 3.262 & 31.688 & 5.275 \\
\hline $\begin{array}{l}\text { Total de likes en } \\
\text { publicaciones }\end{array}$ & 23.446 & 2672 & 9.200 & 255 \\
\hline $\begin{array}{l}\text { Media de me } \\
\text { gusta/publicación }\end{array}$ & 244,22 & 86,19 & 209,09 & 19,61 \\
\hline $\begin{array}{l}\text { Total de comentarios en } \\
\text { publicaciones }\end{array}$ & 541 & 99 & 255 & 15 \\
\hline $\begin{array}{l}\text { Media de } \\
\text { comentarios/publicación }\end{array}$ & 5,63 & 3,19 & 5,79 & 1,15 \\
\hline $\begin{array}{l}\text { Total de compartidos en } \\
\text { publicaciones }\end{array}$ & 6.059 & 474 & 1972 & 26 \\
\hline $\begin{array}{l}\text { Media de } \\
\text { compartidos/publicación }\end{array}$ & 63,11 & 15,29 & 44,81 & 2 \\
\hline \multicolumn{5}{|c|}{ Twitter } \\
\hline & Compromis & Ciudadanos & M. Oltra & C. Punset \\
\hline Seguidores & 22.395 & 5.594 & 88.399 & 8.568 \\
\hline Total de tuits & 2.137 & 2.723 & 2.382 & 457 \\
\hline Media de retuits/tuit & 13,19 & 20,62 & 35,02 & 50,77 \\
\hline Total de retuits & 995 & 1.493 & 1.732 & 380 \\
\hline Media de favoritos/tuit & 6,14 & 11,31 & 25,47 & 42,22 \\
\hline
\end{tabular}

Fuente: elaboración propia 


\section{CONCLUSIONES}

A partir de los resultados expuestos en el epígrafe precedente, se observa que, en términos generales, se ha hecho uso de los entornos 2.0 en el transcurso de la campaña de las primarias, con lo que queda confirmada la $\mathrm{H} 1$, salvo en lo relativo al uso de la web por parte de Ciudadanos. Esta cuestión se presenta debido a la ausencia de Web propia del Partido a nivel autonómico y de información relativa a las primarias en la Web del Partido a nivel nacional.

No obstante, hay un uso desigual de los perfiles analizados en relación al resto de las hipótesis planteadas como eje de la presente investigación.

En primer lugar, queda patente que, en el caso de Compromís, se puede confirmar la $\mathrm{H} 2$ al ser empleados los medios sociales como canal para generar y difundir información en torno a las primarias, dar su apoyo a la candidata en el proceso, y ser vía para pedir la participación. En el caso de Ciudadanos, se confirma la hipótesis en lo relativo a la información sobre primarias y a la promoción de la candidata, si bien se concentra fundamentalmente en el día de su proclamación, pero no hay petición de participación alguna.

En segundo lugar, los resultados permiten confirmar la $\mathrm{SH} 2.1$ al hallar un promedio superior de publicaciones y tuits en Ciudadanos respecto a Compromís, dada la posibilidad de lanzar mayor número de mensajes diarios sin el peligro de sobrecargar de información al seguidor, a la luz de la Gráfico 1.

En tercer lugar, la $\mathrm{H} 3$ viene avalada por los resultados mostrados en el Gráfico 2, en lo relativo a frecuencia de actualización de los respectivos perfiles en Facebook y Twitter. Respecto al contenido, también queda comprobada la H3 en los Gráficos 5 y 6.

En cuarto lugar, se puede confirmar la SH3.1 parcialmente al calcular la media de mensajes por día, resultando más proactivo el perfil de Oltra en Twitter pero no en Facebook, donde lo es Punset por una diferencia mínima (1,22 frente a 1,44 mensajes respectivamente).

En quinto y último lugar, se confirma parcialmente la SH3.2. Paradójicamente, la parcialidad de la misma, deriva de una mayor interacción social a través del perfil en Facebook de Mónica Oltra sin encontrar tal realidad en Twitter, red en la cual Punset presenta un promedio de respuesta ciudadana superior.

En conclusión, parece confirmarse una tendencia al uso de las redes sociales en periodos de campaña (en este caso, primarias) a fin de generar contenido propio que pueda ser difundido allende sus seguidores, facilitando con ello la ampliación del impacto de cada mensaje y el feedback con el ciudadano-usuario. Si bien no puede olvidarse la impronta de la brecha digital y que una parte de la población carece de perfil en las redes, es también destacable cómolos simpatizantes, afiliados y militantes pueden sentirse más protagonistas de la campaña y del propio proceso de democracia interna. Las herramientas 2.0 bien empleadas pueden acercar al Partido y a la candidata al ciudadano, permitiéndoles no solo mostrar el proceso de primarias sino involucrar a los propios y ajenos en el mismo. A pesar de ello, su uso todavía tiene margen de mejora y ello debería observarse en las elecciones de mayo de 2015.

\section{REFERENCIAS BIBLIOGRÁFICAS}

ABEJÓN, P; SASTRE, A; Y LINARES, V.: "Facebook y Twitter en campañas electorales en España." Anuario Electrónico de Estudios en Comunicación Social Disertaciones, Vol. 5(2012), n. 1, pp: 130-159. Disponible en: http://erevistas.saber.ula.ve/index.php/Disertaciones [Fecha de consulta:27 de marzo de 2015]

ARAGÓN, P.; KAPPLER, K.; KALTENBRUNNER, A.; LANIADO, D.; VOLKOVICH, Y: "Communication dynamics in twitter during political campaigns: The case of the 2011 Spanish national election". Policy\& Internet, $\mathrm{n}^{\circ} 5$ (2013), pp: 183-206. 
CALDEVILLA, D. (2009):“Democracia 2.0: la política se introduce en las redes sociales”. Pensar la Publicidad, 3 (2), pp: 31-48.

CASTELLS, M. (2010): “La democràcia en l'erad'Internet”. Revista del Centre d'Estudis Jordi Pujol, n 12, pp: 713.

CENTRO DE INVESTIGACIONES SOCIOLÓGICAS (2015): "Preelectoral elecciones autonómicas y municipales 2015. Comunidad Valenciana". Estudio n 3066 (marzo/abril 2015). Disponible en: http://datos.cis.es/pdf/Es3066mar_A.pdf [Fecha de consulta: 30 de marzo de 2015]

CONGOSTO, M.L. (2015): “Elecciones europeas 2014: viralidad de los mensajes en Twitter”. Revista Hispana para el análisis de Redes Sociales, Vol. 26, n 1, pp: 23-52

CORRADO, A. y FIRESTONE C. M. (eds). (1997): Elections in Cyberspace: Toward a New Era in American Politics, Washington DC: The Aspen Institute.

DE UGARTE, D. (2007):El poder de las redes:Manual ilustrado para personas, colectivos y empresas abocados al ciberactivismo. Barcelona: El Cobre Ediciones.

GARCÍA, E.: "Revoluciones 2.0, redes sociales y política. Reflexiones en torno a \#Primaveravalenciana y \#Ardevalencia”. En: AZNAR, H., MARCO, J. y SANCHO, E. (edit.).(2012).La Política del siglo XXI: ¿Más o menos ciudadanía? Valencia: Tirant Humanidades, pp: 89-96.

INSTITUTO NACIONAL DE ESTADÍSTICA (2014):“Encuesta sobre Equipamiento y Uso de Tecnologías de Información y Comunicación en los Hogares". Disponible en: http://www.ine.es/prensa/np864.pdf[Fecha de consulta: 30 de marzo de 2015]

LÓPEZ, G. LARA, T. y SÁNCHEZ-DUARTE, J.M. "Los weblogs como mecanismos de reafirmación ideológica: la globosfera española ante las elecciones generales de 2008”. En: SAMPEDRO, V. (Coord.).

(2011)Cibercamaña. Cauces y diques para la participación. Madrid: Editorial Complutense, pp: 201- 236.

MERINO, M., LLOVES, B., PÉREZ, A. M.(2013):“La interacción de los usuarios en los perfiles de Facebook de la prensa española”, Palabra Clave,16 (3), pág. 842-872.

NICASIO, B. y PÉREZ, M. (2015): "Iniciativas ciudadanas 2.0 en torno a la elaboración y aprobación de la ley de transparencia". En: MARCO, J. y NICASIO, B. (coord.), La Regeneración del Sistema: reflexiones en torno a la calidad democrática, el buen gobierno y la lucha contra la corrupción, Olelibros, pp: 131-146. Disponible en:http://avapol.es/documentos/La-Regeneracion-del-Sistema.PDF

PEYTIBI, X., RODRÍGUEZ, J.A., Y GUTIERREZ-RUBí, A. (2008): "La experiencia de las elecciones generales de 2008", Revista de Internet, Derecho y Política, nº.7, pp: 26-37.

REY, J. (2007): Comunicación Política, Internet y Campañas Electorales: de la teledemocracia a la ciberdemocracia. Madrid: Tecnos

RODRÍGUEZ, R. y UREÑA, D.:"Diez razones para el uso de Twitter como herramienta en la comunicación política y electoral". Comunicación y Pluralismo, Vol. 10(2011), pp: 89-116.

SAMPEDRO, V., LÓPEZ, J.A. y MUÑOZ, C.: "Ciberdemocracia y cibercampaña: ¿un matrimonio difícil? El caso de las elecciones generales en España en 2008”.ARBOR. Ciencia, Pensamiento y Cultura, Vol 188-756 (julioagosto 2012),pp:657-672.

SAMPEDRO, V., SÁNCHEZ, J.M. y POLETTI, M.: "Ciudadanía y Tecnopolítica Electoral. Ideales y límites burocráticos a la participación digital”. Co-herencia, Vol. 10 (2013), n¹8, pp: 105-136.

TÚÑEZ, M. Y SIXTO, J.: "Redes sociales, política y Compromiso 2.0: "La comunicación de los diputados españoles en Facebook”. Revista Latina de Comunicación Social, nº 66 (2011),pp: 210- 246. Disponible en: http://issuu.com/revistalatinadecomunicacion/docs/rlcs_art930[Fecha de consulta:27 de marzo de 2015] 
VERGEER, M., HERMANS, L. y SAMS, S.:"Online social networks and microblogging in political campaigning: The exploration of a new campaign tool and a new campaign style".PartyPolitics, Vol. $19, n^{\circ} 3$, pp: 477 501Disponible en: http://ppq.sagepub.com/content/early/2011/06/16/1354068811407580.abstract [Fecha de consulta: 27 de marzo de 2015]

\section{BREVE SEMBLANZA DE LAS AUTORAS:}

Marta Pérez Gabaldón es Licenciada en Ciencias Políticas y de la Administración por la CEU-Cardenal Herrera, y Doctora por la misma Universidad, obteniendo en ambos casos premio extraordinario. En la actualidad, es profesora en los Grados de Derecho, Periodismo y Ciencias Políticas, y ocupa la Secretaría Académica de la Facultad de Derecho, Empresa y Ciencias Políticas UCH-CEU.

Blanca Nicasio Varea es Licenciada en Periodismo (Premio Extraordinario) y en Ciencias Políticas y de la Administración por la Universidad CEU Cardenal Herrera. En septiembre de 2011 obtuvo el Diploma de Estudios Avanzados y, en la actualidad, disfruta de una Beca de Formación de Profesorado Universitario del Ministerio de Educación, Cultura y Deporte.

[1] La Encuesta tiene periodicidad anual. Las entrevistas se realizan entre enero y mayo del año en curso por teléfono o mediante visita personal.

[2] @monicaoltraenTwittter y Mónica Oltra en Facebook

[3]@carolinaPunset en Twitter y Carolina PunsetBannel en Facebook

[4] @compromis y @CsCValenciana en Twitter, y Ciudadanos C’s Valencia y Coalició Compromís en Facebook

[5] De un censo de 39.650 electores inscritos, participaron 24.707 (62,31\% del censo), dándole su apoyo 22.152 (el $55,8 \%$ del censo)

[6] 25 de marzo de 2015

Ámbitos. Revista Internacional de Comunicación, n.29, año 2015, tercer trimestre (verano).

Recibido: 02/05/2015

Aprobado: 25/06/2015 